\title{
Definición de consumo de hombres universitarios. Exploración desde las redes semánticas naturales
}

\author{
Definition of university men's consumption. Exploration from
}

\section{natural semantic networks}

Loreto Arias Lagos ${ }^{1}$, Marianela Denegri Coria ${ }^{2}$ y Felipe Sáez Ardura ${ }^{3}$

${ }^{1}$ Doctorante en Ciencias Sociales. Universidad de La Frontera (UFRO). Temuco, Chile. Becaria ANID-PFCHA/Doctorado Nacional/2018-21180055. E-mail: loreto.arias@gmail.com ${ }^{2}$ Doctora en Psicología. Directora del Centro de Excelencia en Psicología Económica y del Consumo y Directora del Núcleo Científico Tecnológico en Ciencias Sociales y Humanidades, Universidad de La Frontera (UFRO). Temuco, Chile. E-mail: marianela.denegri@ufrontera.cl ${ }^{3}$ Doctor en Ciencias Sociales. Docente de la Universidad Autónoma de Chile. Temuco, Chile.

E-mail: inti.felipe@gmail.com

Universidad de La Frontera. Núcleo Científico Tecnológico en Ciencias Sociales y Humanidades. Centro de Excelencia en Psicología Económica y del Consumo (CEPEC)

Temuco, Chile

\section{Resumen}

Frente al dominio del neoliberalismo en las sociedades contemporáneas, las posibilidades de consumo resultan abrumadoras, en tanto mecanismo de configuración identitaria $\mathrm{y}$ evidencia de nuevas formas de inclusión/ exclusión de los sujetos respecto de los grupos sociales. Esta investigación aborda un segmento de la sociedad chilena que no escapa de esta lógica: los hombres jóvenes. Los jóvenes, en general, constituyen un segmento propenso al riesgo financiero, con altos niveles de endeudamiento, al menos en la sociedad chilena. En este escenario, el artículo explora los significados de consumo que construyen hombres universitarios de pregrado, de tres áreas de formación profesional de una universidad de la Región de La Araucanía (Chile). Se utiliza una metodología cualitativa, aplicando la técnica de redes semánticas naturales. La muestra estuvo conformada por estudiantes de las áreas de las Ciencias Sociales, Ciencias de la Salud y Ciencias de la Ingeniería, de edades entre 18 y 24 años, con un total de 51 sujetos. Los resultados indican una red semántica general abundante, cuyo núcleo está representado por la palabra "alimentarse", seguido por significados asociados a dinero, comprar y necesidad, que dan cuenta de la dimensión material del consumo. Son relevantes, además, los conceptos asociados a drogas y alcohol. A propósito de los hallazgos, se sugiere explorar la relación de la alimentación con el cuerpo masculino, así como la posesión de bienes materiales y las orientaciones al éxito en los hombres jóvenes, sobre todo en lo referente al proceso de construcción de masculinidades juveniles.

Palabras clave: consumo, sociedad de consumo, hombres universitarios de pregrado, jóvenes, red semántica natural.

\section{Abstract}

In the face of the dominance of neoliberalism in Western societies the possibilities 
of consumption are overwhelming. This type of societies are associated with a consumerist culture and lifestyle, where the capacity of consumption is the fundamental criterion of inclusion/exclusion of the subjects. Consumption intervenes significantly in the construction of people's personal and social identity. The desires and longings generated by the consumer society are related to the acquisition of lifestyles. In this sense, subjects give meaning to objects and experiences. The interest of this research is in young people. Young people represent a segment of interest for the economic market; therefore, they constitute a group that is financially in risk. The high levels of indebtedness of the youth population in Chile must be considered. Specifically, men handle money from a young age and carry out certain paid activities, especially in popular sectors. Men are projected as active consumers in the economic market, an area where the traditional gender division has diminished, directing men to production and women to consumption. On the other hand, empirical evidence shows the association between masculinities and material possessions, money and orientation to success. It is also possible to mention a relative scarcity of studies linking men and consumption in Latin America. In this context, the present article explores the meaning of consumption as constructed by university men in three areas of professional training, who are pursuing undergraduate careers, in the Araucanía Region. A qualitative methodology is used to achieve the research objective, applying the technique of natural semantic networks. A case study is carried out. The sample was conformed by students of Social Sciences (18 subjects), Health Sciences (15 subjects) and Engineering Sciences (18 subjects), aged between 18 and 24 years old, with a total of 51 subjects. All students are from a private university in the Araucanía Region, Chile.

From the study carried out, it is possible to point out the usefulness of the technique of natural semantic networks as a tool for a first exploration of the field of study. The results indicate an abundant general semantic network, formed by 129 words. The core of the semantic network is represented by the word "feed", followed by meanings associated with money, buying (shopping) and need, giving an account of the material dimension of consumption. The definitions associated with drugs and alcohol are also relevant. For Social Science students, the SAM net for consumption is defined by money, expenses and need. The SAM net for young men in Health Sciences is defined by food, money and drugs. For engineering students, consumption is defined by expenses, food and money. In summary, the results of the overall SAM net indicate three major categories of exploration of meanings relevant to undergraduate college youth consumption in this study: food and eating; appropriation of material possessions in relation to acquisition and exchange for money from purchase, and consumption associated with drugs and alcohol. The findings suggest primarily exploring food and the relationship with the male body, as well as material possessions and orientations to success in young men and the relationship with the construction of youthful masculinities.

Keywords: consumption, consumer society, undergraduate male students, young people, natural semantic network.

\section{Introducción}

El consumo es parte de la vida cotidiana de todos los seres humanos. Parte integral de la supervivencia biológica de los sujetos, a través de la historia, las tareas relacionadas al consumo (producción, almacenamiento, distribución y eliminación de objetos de consumo) se han configurado como modeladoras de las formas de vida de los sujetos en las sociedades humanas, de tal manera que se incrustan en el seno de las relaciones sociales (Bauman, 2016). Hablar de consumo no es circunscribirse solo a la 
satisfacción de necesidades o a las formas de comportamiento individual respecto de los bienes y servicios, sino que es mucho más que eso: en las sociedades posmodernas, se considera a sus miembros en calidad de consumidores (Baudrillard, 2007; Bauman, 2014, 2016). Así, lo que determina esta sociedad es el rol central del consumo en la vida de los sujetos. Esto conlleva a una vida guiada por anhelos inagotables de adquirir y usar objetos, en la que no se asocia tanto la felicidad con la gratificación, sino que más bien "con un aumento permanente del volumen y la intensidad de los deseos" (Bauman, 2016, p. 50). Dado ello, en las sociedades de consumidores se juzga y evalúa a sus miembros sobre todo por "sus capacidades y su conducta en relación con el consumo" (Bauman, 2014, p. 110). Si bien el consumidor es más libre en el ámbito privado, depende más del mercado para satisfacer sus deseos. Para Lipovetsky (2007), la fase del hiperconsumo se caracteriza por un consumo para sí, en la que los gastos terminan dando cuenta de una personalización del consumo orientado a la búsqueda de la felicidad privada y la optimización de los recursos corporales y comunicativos.

A partir de lo anterior, es preciso considerar que el consumo interviene de manera significativa en la construcción de la identidad personal y social de las personas, de modo que los bienes constituyen una expresión de aquello (Bauman, 2016; Lipovetsky 2007; Sassatelli, 2012), por lo que pasa a ser una prolongación de la imagen de uno mismo (Denegri et al., 2014). En este escenario, los deseos y anhelos generados por la sociedad de consumo se relacionan con la adquisición de estilos de vida, de manera tal que adquieren significación simbólica en la vida de los sujetos (Huber, 2002). Esto es particularmente relevante en los jóvenes, ya que es en la adolescencia cuando se consolida la identidad y los patrones de comportamiento que persistirán en la vida adulta (Barros et al., 2015). Específicamente en la etapa de los 18 a 25 años, es importante explorar la identidad, considerada una fase heterogénea e inestable, así como también un umbral entre el mundo adolescente y el adulto (Arnett, 2007, 2015; Connell, 2003).

El consumo configura un estado de bienestar material y esto ha tenido implicancias positivas para la sociedad (Lipovetsky, 2007; Lipovetsky y Serroy, 2010), no obstante, también son evidentes las contradicciones. Araujo y Martuccelli (2012) señalan que la universalidad de las expectativas de acceso a bienes de la población chilena ha generado un sentimiento inédito de pertenencia a un colectivo nacional. Este sentimiento, a su vez, se acompaña de segmentación, insatisfacción -al no poder comprar- $\mathrm{y}$ problemas de endeudamiento, lo que acentúa considerablemente el riesgo financiero en poblaciones juveniles, carentes de educación financiera, y que por sus características de desarrollo están más expuestas a las presiones del mercado (Castellanos et al., 2016). Esto se aprecia a partir de las cifras que develan el nivel de endeudamiento en los jóvenes chilenos, el cual se incrementa en función de la edad: un $4 \%$ en el rango de 15 a 19 años, un $36 \%$ en el de 20 a 24 años, y un $55 \%$ en el de 25 a 29 años (INJUV, 2017).

La evidencia muestra que los jóvenes, en el contexto chileno, perciben una mejor situación material que sus padres, e internalizan las exigencias económicas del sistema neoliberal (Álvarez y Garcés, 2017). De hecho, jóvenes y niños son objetivo de variadas estrategias de mercado, quienes en su mayoría no tienen renta propia, pero sí una notoria capacidad de endeudamiento y de influencia dentro de las decisiones económicas de familia (Barros et al., 2015).

Estudios en jóvenes de la generación $\mathrm{Y}$ (o millennials), que corresponden mayoritariamente a estudiantes universitarios y primeros trabajadores, hablan de una generación de jóvenes muy orientada al consumo por la masificación del uso de las tecnologías de la comunicación, los medios de 
comunicación e internet (Bakewell y Mitchell, 2003), proclive al consumismo y a definir su identidad por sus compras o experiencias (Kim y Jang, 2014). Así, el consumo favorece la autorrealización y el sentimiento de inclusión a grupos de pares (PNUD/INJUV, 2003), en el marco de una cultura material inclinada a internalizar valores de consumo en grupos vulnerables (Dittmar, 2011). En los varones de bajos ingresos, estas formas de consumo reafirmarían masculinidades; para las cuales no estar directamente en sintonía con cánones modernos de consumo generaría exclusión (Rosenmann et al., 2017); lo que esto afecta, incluso, factores psicosociales como el autoconcepto, la autoestima o las actitudes hacia lo masculino (Maguire y Stanway, 2008; Isaksen y Roper, 2008, 2012).

En Chile, un estudio con adolescentes de mediados de la década pasada muestra que, entre las alternativas de compra más declaradas, están los alimentos (snacks y golosinas), con un $82 \%$; luego la música y la tecnología, con un $28 \%$; y la ropa, el maquillaje y los accesorios, con un $26 \%$ (CNTV y McCann Erikson, 2005). Otro estudio (Pallavicini, 2008) describe la centralidad que los objetos de consumo alcanzan en los procesos de individuación de los adolescentes y cómo la personalización opera mediante el consumo de objetos en serie y de moda, en la el que intervienen condiciones como la estructura socioeconómica y el género. Por ejemplo, para los hombres, las marcas son relevantes en las decisiones de compra, ya sea por calidad o por estatus; y la estética -verse bien- es la motivación central en las elecciones de consumo de las mujeres. Por otra parte, Duarte (2009) reporta el consumo opulento como mecanismo de mayor ocurrencia para lograr el éxito en jóvenes empobrecidos, hecho que se relaciona con la inserción laboral eficiente como vía para el acceso al consumo y la modernización tecnológica como instrumento de prestigio y valoración.

Para el espacio regional, estudios empíricos como los de Barros et al. (2015) y Denegri et al. (2017) indagan en las prácticas de consumo de adolescentes urbanos de la Región de La Araucanía (Chile) en los que y concluyen que ellos ocupan su dinero en gastos personales, ocio y alimentación, y presentan prácticas de endeudamiento mediante préstamos a redes de apoyo cercanas, ya que no comprar implica frustración y resignación (Barros et al., 2015).

Un estudio sobre adultos jóvenes en Colombia (Ortega y Rodríguez, 2005) identifica orientaciones a hábitos reflexivos de consumo, con una elevada proporción de sujetos que consideran el crédito como una parte esencial del estilo de vida actual (78 \%). Denegri et al. (2014), en una investigación con jóvenes estudiantes universitarios de dos universidades del sur de Chile, muestran que el consumo juega un papel importante en la construcción del concepto del Yo, lo que entrega a los sujetos una materialización concreta de la consecución de sus aspiraciones. Asimismo, Godoy et al. (2018) dieron cuenta de niveles favorables de actitudes hacia el ahorro, hacia el endeudamiento y hacia la racionalidad en la compra en universitarios recientemente titulados.

Otras investigaciones incluyen la variable género en el campo de estudios sobre consumo en jóvenes. González et al. (2016) muestran que el consumo se constituye principalmente como una actividad de ocio feminizada. Sin embargo, los varones se han incorporado gradualmente al acto de comprar y no están de acuerdo con la asociación consumo-derroche. Botero et al. (2008) encontraron altos índices de consumo simbólico en hombres y mujeres universitarios de Colombia, donde el consumo no es únicamente motivado por la utilidad de los objetivos. Además, las jóvenes presentan mayores índices de consumo compulsivo y los varones, mayores niveles de materialismo en la compra. Esto coincide con los resultados reportados por Barros et al. (2019), en un estudio con adolescentes rurales al sur de Chile, en el que los hombres otorgan mayor valor a las posesiones materiales, y asocian el dinero con la felicidad y con expectativas laborales 
altamente remuneradas. Considerando la masculinidad de adultos jóvenes, Eastman et al. (2018) mostraron que la masculinidad media la relación entre el consumo orientado por el estatus y la intención de compra, en losa que la masculinidad estaría relacionada con la orientación al éxito, el dinero y las posesiones (Eng y Bogaert, 2010; Hofstede, 1980; Kim y Jang, 2014; Olavarría, 2018; Weidmann et al., 2009, citado en Eastman et al., 2018). Es decir, el consumo de bienes se ha reportado como un mecanismo valorado por jóvenes universitarios (Cubilllas et al., 2016).

Como muestran los hallazgos presentados, la investigación en torno al consumo y los jóvenes cobra relevancia en sociedades como la chilena, en la que el modelo neoliberal ha tenido un desarrollo exitoso. Considerando, entonces, que "la cultura juvenil gira en torno al consumo" (Sassatelli, 2012, p. 121), es posible advertir, en el caso de los varones particularmente, que estos desde la infancia manejan dinero con mayor prevalencia que las mujeres y pueden comenzar a desarrollar actividades remuneradas desde más pequeños; los sectores populares son un espacio claro de aquello (Olavarría, 2000). Las cifras para Chile respaldan esta tendencia, ya que el 39 $\%$ de las mujeres recibe ingresos producto de su trabajo, ya sea regular o esporádico, mientras que en el caso de los hombres se alcanza un $58 \%$ (INJUV, 2017). Asimismo, interesa la relación entre el género masculino y el consumo, relación que se ha estudiado en menor medida (Belk y Costa, 1998; González et al., 2016; Littlefield y Ozanne, 2011), como se ha podido evidenciar con la revisión de literatura relativa a la temática en América Latina.

La socialización de género reproduce desigualdades en relación con roles de género, de manera que impacta, incluso, el acto de comprar (González et al., 2016); lo que relativiza la tradicional división de género (Alexander, 2003; Borrás, 2008; Connell, 1997; González et al., 2016), y proyecta ahora a los hombres jóvenes como activos consumidores. A partir de los antecedentes expuestos, el objetivo de esta investigación es explorar los significados asociados al consumo en un segmento social emergente en Chile, el de los hombres universitarios de pregrado de tres áreas de formación profesional (Ciencias Sociales, Ciencias de la Salud y Ciencias de la Ingeniería) de una universidad privada de la Región de La Araucanía (Chile). Este segmento ha logrado expandir su matrícula en Chile en un $60 \%$ durante los últimos 20 años (Pérez, 2014), y ha alcanzado las 442 675 matrículas ya a mediados de la década pasada y las 668854 en 2019 (CNED, 2019). En particular, la Región de La Araucanía en el año 2019 contó con 35520 estudiantes (CNED, 2019), cifra creciente en comparación con décadas anteriores. Específicamente, se busca: (1) identificar los conceptos emergentes asociados a los significados de consumo; (2) identificar la relevancia de los conceptos emergentes asociados a los significados de consumo; y (3) identificar diferencias y similitudes en los conceptos emergentes asociados a los significados de consumo según el área profesional.

A propósito de la escasez de estudios que relacionen hombres y consumo en países latinoamericanos, se utilizan las redes semánticas como primera aproximación al tema de estudio, lo que es útil cuando existen dudas respecto de los significados de un grupo sobre un objeto (Vera et al., 2005). Esta técnica, desarrollada por Figueroa, González y Solís (1981) y, posteriormente, por Valdez (1998), retoma algunos de los postulados de la psicología cognitiva respecto de la memoria semántica, para aproximarse al significado que los individuos construyen de un concepto. En este contexto, el significado "es la unidad fundamental de la organización cognoscitiva, que está compuesta de elementos afectivos y de conocimiento, que crean un código subjetivo de reacción" (Valdez, 1998, p. 50). Esto supone una organización interna de la información contenida en la memoria a largo plazo, en forma de red, en la que las palabras 
forman relaciones que engloban el significado de un concepto. Así, a través del lenguaje es posible conocer y comprender los procesos mentales, facilitado por la íntima relación entre el lenguaje, el pensamiento, la percepción y el aprendizaje (Chomsky, 1977). El significado psicológico, aquí, depende del contexto específico de los sujetos, por lo cual rescata aspectos culturales y sociales imbricados en su vida, y se construye "una unidad de presentación cognoscitiva y de estimulación para la producción de la conducta" (Valdez, 1998, p. 56).

\section{Método}

Se ha desarrollado un estudio exploratorio y descriptivo, de corte cualitativo. La estrategia usada corresponde a un estudio de caso que indaga, mediante uno o más casos, un contexto particular (Creswell, 2007), que en esta oportunidad atañe al consumo. Específicamente, se utilizó un estudio de caso único, en el que se considera como sujetos a jóvenes varones de una universidad privada ubicada en la ciudad de Temuco, capital de la Región de La Araucanía. La universidad en cuestión se encuentra adscripta al sistema de gratuidad, que beneficia al $60 \%$ más pobre de la población considerando el arancel gratuito y la matrícula de la carrera cursada durante los años formales de duración (MINEDUC, 2019).

\section{Participantes}

El muestreo utilizado fue de tipo intencional (Ruiz, 2012), y el criterio para la elección de los sujetos implicó la heterogeneidad (Valles, 2002). De este modo, la muestra estuvo conformada por estudiantes de las áreas de Ciencias Sociales (18 sujetos), Ciencias de la Salud (15 sujetos) y Ciencias de la Ingeniería (18 sujetos), de entre 18 y 24 años; así alcanzó un total de 51 sujetos. Se buscó, en las definiciones de consumo entregadas por los sujetos, captar una diversidad de significados, en función de los diversos contextos académicos de los estudiantes.

\section{Instrumentos}

La técnica de recolección de información correspondió a las redes semánticas naturales (Figueroa et al., 1981; Valdez, 1998), que busca obtener información del significado de los conceptos que se utilizan como estímulo, desde los individuos, y evitar taxonomías artificiales creadas por los investigadores para explicar la organización de la información referente a la memoria semántica. Esta técnica se acerca al significado psicológico y, con esto, a la construcción de conocimiento de los sujetos (Valdez, 1998), proceso reconstructivo de información que permite acceder al conocimiento que se tiene de un concepto a partir del lenguaje de los sujetos (Figueroa et al., 1981, citado por Valdez, 1998). Además, es posible dar cuenta de la distancia semántica entre los significados que definen un concepto, ya que no todos los elementos tienen la misma importancia para el nodo o concepto central que se busca precisar. En relación con el instrumento, es pertinente mencionar que Valdez (1998) ha validado la técnica para la evaluación del significado psicológico a partir del análisis factorial.

Como parte de los criterios éticos, se utilizó el consentimiento informado, lo que asegura la confidencialidad en el estudio. Para identificar los significados de los jóvenes universitarios respecto al consumo, el cuestionario se diseñó solicitando: (1) datos sociodemográficos (edad, lugar de procedencia y carrera); y (2) palabra-estímulo (que correspondió a "consumo"). Ha sido diseñado para aplicarse de forma anónima, a fin de buscar que los estudiantes tuvieran libertad para anotar sus definiciones y solicitar su respuesta de manera individual en un tiempo de aplicación de no más de 8 minutos (para la identificación de conceptos y jerarquización), con el fin de mantener la atención de los participantes (Valdez, 1998). Las instrucciones incluyeron: 
definir la palabra "consumo", utilizando verbos, adverbios, adjetivos, sustantivos, nombres o pronombres -sin utilizar artículos ni proposiciones, ni tampoco la palabra "consumo"-; instar a anotar como mínimo cinco palabras que definieran el concepto $\mathrm{y}$, una vez completada la lista, enumerar las palabras en orden de importancia del 1 al 5, considerando 1 para la más importante o más cercana a su definición y el 5 para la menos importante o más lejana. Por último, se puso énfasis en que los participantes anotaran solo una palabra por cada una de las cinco líneas dispuestas.

\section{Procedimiento de análisis}

El procedimiento de análisis se basó en la técnica de redes semánticas naturales, que incluye la realización de las siguientes tareas (Figueroa et al., 1981; Valdez, 1998): (1) determinar el valor J, obtenido al sumar el total de palabras generadas por los sujetos, indicador de la riqueza de la red semántica en la que a mayor número de palabras, mayor riqueza de la red-; (2) determinar el valor $\mathrm{M}$, indicador del peso semántico de cada palabra definitoria de la red, que se consigue multiplicando la frecuencia de aparición por la jerarquía obtenida para cada palabra definitoria otorgada por los sujetos -se mide en unla escala de 1 a 10-; (3) definir el conjunto SAM (Semantic Association Memory), indicador de las palabras definitorias que señalan el núcleo central de la red -en esta oportunidad, la representan las 15 palabras definitorias que obtuvieron los mayores valores $\mathrm{M}$ totales, siguiendo la indicación de Valdez (1998)-; y (4) determinar el valor FMG, indicador en porcentajes de la distancia semántica que existe entre las distintas palabras definitorias del conjunto SAM, valores que se obtienen a partir de una regla de tres que toma como punto de arranque la palabra definitoria con el M más grande, el cual representa el $100 \%$.

\section{Resultados}

La Tabla 1 muestra el conjunto SAM general de los informantes para la palabra "consumo". Se observan las 15 palabras definitorias con mayor valor semántico, que definen el concepto. También se presenta su distancia semántica (valor FMG), generada por el grupo total de estudiantes. El valor J, por su parte, indica la riqueza de la red semántica para la palabra "consumo", que en este análisis es de 129 palabras. El núcleo de la red es la palabra "alimentarse" (100\%), seguido por "gastos" (80 \%), "dinero" (77 \%), "comprar" (56 \%) y "necesidad" (52\%). Luego, están presenten palabras tales como "drogas" (36 \%), "alcohol" (33\%), "dependencia" (33\%) y "tomar"/"beber" (26\%). Posteriormente, aparece la palabra "productos" $(25 \%)$, seguida por "vicios" (21\%), "bienes" (20\%), "ropa" (20\%), "innecesario" (17\%) y "satisfacción" $(17 \%)$.

Tabla 1

Conjunto SAM general para consumo de hombres universitarios.

\begin{tabular}{c|c|c} 
Palabra definitoria & $\begin{array}{c}\text { Valor M } \\
\text { (peso semántico) }\end{array}$ & $\begin{array}{c}\text { Valor FMG (distancia } \\
\text { semántica) (\%) }\end{array}$ \\
\hline Alimentarse & 163 & 100 \\
Gastos & 130 & 80 \\
Dinero & 126 & 77 \\
Comprar & 92 & 56 \\
Necesidad & 84 & 52 \\
Drogas & 59 & 36
\end{tabular}


Arias Lagos, Denegri Coria y Sáez Ardura

\begin{tabular}{c|c|c} 
Palabra definitoria & $\begin{array}{c}\text { Valor M } \\
\text { (peso semántico) }\end{array}$ & $\begin{array}{c}\text { Valor FMG (distancia } \\
\text { semántica) (\%) }\end{array}$ \\
\hline Alcohol & 53 & 33 \\
Dependencia & 53 & 33 \\
Tomar/Beber & 43 & 26 \\
Productos & 41 & 25 \\
Vicios & 35 & 21 \\
Bienes & 33 & 20 \\
Ropa & 33 & 20 \\
Innecesario & 28 & 17 \\
Satisfacción & 27 & 17
\end{tabular}

Fuente: elaboración propia.

La Tabla 2 señala las 15 palabras definitorias con mayor valor semántico (conjunto SAM), que definen el concepto de consumo de los jóvenes estudiantes de Ciencias Sociales, Ciencias de la Salud y Ciencias de la Ingeniería, es decir, diferenciados por área de formación profesional. Para los estudiantes de Ciencias Sociales, el valor $\mathrm{J}$ indica la riqueza de la red semántica para la palabra "consumo", que en este caso está conformado por 61 palabras. El núcleo de la red es la palabra "dinero" (100 $\%)$, seguido por "gastos" (86\%), "necesidad" (76 \%), "compras" (60\%), "alimentarse" (57 \%), "productos" (43\%), "bienes" (41 $\%)$ y "dependencia" (41\%). Conjuntamente, los valores más bajos de la red incluyen las palabras "vicios" (34\%), "tiempo" (28\%), "servicios" (28\%), "ocio" (26\%), "drogas" (22\%), "carrete"1 (22\%) y "todo" (17\%).

Se presentan las 15 palabras definitorias de hombres jóvenes estudiantes de Ciencias de la Salud para el concepto "consumo". El indicador de la riqueza semántica de la red, correspondiente al valor $\mathrm{J}$, en este caso, es de 45 palabras. El núcleo de la red es el concepto "alimentarse" (100\%), lo sigue con una distancia semántica considerable la palabra "dinero" (49 \%). Luego, aparecen las palabras "drogas" (44\%), "alcohol" (44 \%), "tomar"/“beber" (36 \%), "comprar" (34 \%), "ropa" (31\%), "necesidad" (29\%) y "productos" (23\%). Los últimos lugares de la red son ocupados por las palabras "comercio" (24\%), "gastos" (23\%), "vanidad" (20\%), "negocio" (19\%), "satisfacción" (14\%) y "vital" (14\%).

Por último, se hace mención al conjunto SAM para la palabra "consumo" de los estudiantes de Ciencias de la Ingeniería. El indicador de la riqueza semántica de la red corresponde a 59 palabras (valor J). El núcleo de la red lo representa la palabra "gastos" (100\%), seguido de cerca por "alimentarse" (94 \%). Luego, con una distancia más importante aparece "dinero" (53\%), seguido inmediatamente por "comprar" (52 \%). Un poco más lejos en relación con la distancia semántica se encuentra "dependencia" (45 $\%)$, seguido por "tomar"/"beber" (41 \%), "necesidad" (31 \%), “innecesario" (30 \%), "energía" (28\%), "libertad" (27\%) y "alcohol" (25\%). Los últimos lugares de la red son ocupados por "vicio" (23\%), "drogas" (23\%), “adicción” (20\%) y “marihuana” (19 $\%)$.

${ }^{1}$ En Chile, la palabra “carrete" es un término coloquial utilizado para referirse a la fiesta o parranda. 
Tabla 2

Conjunto SAM para consumo de hombres universitarios según facultades.

\begin{tabular}{|c|c|c|c|c|c|c|c|c|}
\hline & & & Facu & des & & & & \\
\hline Cienc & s Social & & Ciencia & de la Sa & & Ciencias d & la Inger & ería \\
\hline $\begin{array}{c}\text { Palabra } \\
\text { definitoria }\end{array}$ & $\begin{array}{l}\text { Valor } \\
\text { M* }^{*}\end{array}$ & $\begin{array}{c}\text { Valor } \\
\text { FMG** }^{* *} \\
(\%)\end{array}$ & $\begin{array}{c}\text { Palabra } \\
\text { definitoria }\end{array}$ & $\begin{array}{c}\text { Valor } \\
\mathrm{M}\end{array}$ & $\begin{array}{c}\text { Valor } \\
\text { FMG } \\
(\%)\end{array}$ & $\begin{array}{c}\text { Palabra } \\
\text { definitoria }\end{array}$ & $\begin{array}{c}\text { Valor } \\
\mathrm{M}\end{array}$ & $\begin{array}{c}\text { Valor } \\
\text { FMG } \\
(\%)\end{array}$ \\
\hline Dinero & 58 & 100 & Alimentarse & 70 & 100 & Gastos & 64 & 100 \\
\hline Gastos & 50 & 86 & Dinero & 34 & 49 & Alimentarse & 60 & 94 \\
\hline Necesidad & 44 & 76 & Drogas & 31 & 44 & Dinero & 34 & 53 \\
\hline Comprar & 35 & 60 & Alcohol & 31 & 44 & Comprar & 33 & 52 \\
\hline Alimentarse & 33 & 57 & Tomar/beber & 25 & 36 & Dependencia & 29 & 45 \\
\hline Productos & 25 & 43 & Comprar & 24 & 34 & Tomar/beber & 26 & 41 \\
\hline Bienes & 24 & 41 & Ropa & 22 & 31 & Necesidad & 20 & 31 \\
\hline Dependencia & 24 & 41 & Necesidad & 20 & 29 & Innecesario & 19 & 30 \\
\hline Vicios & 20 & 34 & Comercio & 17 & 24 & Energía & 18 & 28 \\
\hline Tiempo & 16 & 28 & Gastos & 16 & 23 & Libertad & 17 & 27 \\
\hline Servicios & 16 & 28 & Productos & 16 & 23 & Alcohol & 16 & 25 \\
\hline Ocio & 15 & 26 & Vanidad & 14 & 20 & Vicio & 15 & 23 \\
\hline Drogas & 13 & 22 & Negocio & 13 & 19 & Drogas & 15 & 23 \\
\hline Carrete & 13 & 22 & Satisfacción & 10 & 14 & Adicción & 13 & 20 \\
\hline Todo & 10 & 17 & Vital & 10 & 14114 & Marihuana & 12 & 19 \\
\hline
\end{tabular}

Notas: *corresponde al peso semántico; **corresponde a la distancia semántica.

Fuente: elaboración propia.

La Tabla 3 agrupa las cinco palabras definitorias más altas de consumo (con mayor peso semántico) y las cinco palabras definitorias más bajas (con menor peso semántico), con el correspondiente valor FMG, según las áreas de formación profesional. Como es posible observar, el núcleo de la red para los estudiantes es diferente según el área profesional. En el caso de la palabra definitoria "alimentarse", esta se presenta con altos pesos semánticos en los estudiantes de Ciencias de la Salud (100\%), Ciencias de la Ingeniería (94\%) y, en menor medida, en Ciencias Sociales (57 \%).

En el caso de los jóvenes de Ciencias de la Salud, la distancia semántica de la palabra definitoria "dinero" es amplia (49\%), lo que indica un peso semántico importante del núcleo de la red en la definición de consumo de estos jóvenes. La palabra "gastos" se presenta en la definición de consumo de manera relevante en estudiantes de Ciencias de la Ingeniería $(100 \%)$ y Ciencias Sociales (86\%), pero no está presente en las cinco palabras definitorias para consumo de los estudiantes de Ciencias de la Salud. La palabra "dinero", por su parte, se encuentra en la red semántica de consumo en los jóvenes de las 3 áreas, y constituye el núcleo para los jóvenes de las Ciencias Sociales $(100 \%)$. Cabe mencionar que, en el caso de los estudiantes de Ciencias de la Salud, la presencia de palabras definitorias significativas para la palabra "consumo" en relación con el alcohol y las drogas se 
encuentra en los primeros lugares de la red, una diferencia con los estudiantes de las otras áreas, que sitúan estas palabras en los últimos lugares de la red semántica. La centralidad del consumo para los informantes de las Ciencias
Sociales queda de manifiesto en las palabras definitorias "necesidad" (76 \%) y "todo" (17 $\%)$. En el caso de los estudiantes de Ciencias de la Salud, esto se refleja en la palabra definitoria "vital" (14\%).

Tabla 3

Palabras definitorias de alto y bajo peso semántico para consumo de hombres universitarios según facultades.

\begin{tabular}{|c|c|c|c|c|c|c|}
\hline & \multicolumn{6}{|c|}{ Facultades } \\
\hline & \multicolumn{2}{|c|}{ Ciencias Sociales } & \multicolumn{2}{|c|}{ Ciencias de la Salud } & \multicolumn{2}{|c|}{ Ciencias de la Ingeniería } \\
\hline & Alto & Bajo & Alto & Bajo & Alto & Bajo \\
\hline \multirow{5}{*}{$\begin{array}{l}\text { Palabra } \\
\text { definitoria }\end{array}$} & Dinero & Servicios & Alimentarse & Productos & Gastos & Alcohol \\
\hline & Gastos & Ocio & Dinero & Vanidad & Alimentarse & Vicio \\
\hline & Necesidad & Drogas & Drogas & Negocio & Dinero & Drogas \\
\hline & Comprar & Carrete & Alcohol & Satisfacción & Comprar & Adicción \\
\hline & Alimentarse & Todo & Tomar/beber & Vital & Dependencia & Marihuana \\
\hline \multirow{6}{*}{$\begin{array}{c}\text { Valor } \\
\text { FMG }^{*}\end{array}$} & $\%$ & $\%$ & $\%$ & $\%$ & $\%$ & $\%$ \\
\hline & 100 & 28 & 100 & 23 & 100 & 25 \\
\hline & 86 & 26 & 49 & 20 & 94 & 23 \\
\hline & 76 & 22 & 44 & 19 & 53 & 23 \\
\hline & 60 & 22 & 44 & 14 & 52 & 20 \\
\hline & 57 & 17 & 36 & 14 & 45 & 19 \\
\hline
\end{tabular}

Fuente: elaboración propia.

Notas: *corresponde a la distancia semántica

\section{Discusión}

Los resultados dados a conocer representan los significados de consumo para hombres jóvenes de una universidad privada de la Región de La Araucanía. En ese aspecto, la técnica de las redes semánticas naturales se posiciona como una opción para aproximarse a los significados de consumo desde los jóvenes, a partir de una evaluación del significado psicológico y su relación con la cultura (Valdez, 1998). Dado ello, la aplicación de la técnica resulta un ejercicio interesante de exploración del campo de estudio que abre la posibilidad de profundizar en ciertas temáticas en función de la definición de los participantes.

La investigación realizada permite obtener una mirada general del fenómeno del consumo desde los hombres universitarios de un espacio regional. Ahora bien, considerando el conjunto SAM general, es posible identificar tres grandes categorías de exploración propuestas como relevantes en la definición de consumo de los jóvenes universitarios: (1) la alimentación y la comida; (2) la apropiación de bienes por dinero a partir de la compra (adquisición/intercambio); y (3) el consumo asociado a las drogas y el alcohol.

Respecto al conjunto SAM de los hombres 
jóvenes de las Ciencias Sociales, es posible afirmar que, para estos, el consumo se define a partir del dinero y la compra. El gasto es considerado como necesidad $y$, dentro de aquello, los alimentos tienen una relevancia importante. El conjunto SAM de los hombres jóvenes de las Ciencias de la Salud se define por la alimentación, por el dinero y el consumo de alcohol y drogas. Para los hombres jóvenes de las Ciencias de la Ingeniería, por su parte, el consumo se define a partir de los gastos, los alimentos y la compra.

Los resultados del conjunto SAM general para la palabra "consumo" de los jóvenes hombres universitarios que conformaron la muestra dieron cuenta de una nutrida red semántica formada por 129 palabras, lo que lleva a considerar múltiples significados para los informantes. En este sentido, la relevancia de la palabra "alimentarse", núcleo de la red, es coincidente con otros estudios en los que la alimentación es una práctica de consumo relevante en adolescentes y jóvenes (CNTV y McCann, 2005; Denegri et al., 2017). Cabe señalar que existe evidencia de la relación de los hábitos de alimentación con la satisfacción con la propia vida, así como con la satisfacción en relación con su alimentación (Schnettler et al., 2011). Particularmente en este estudio, se destaca la presencia de la palabra "alimentarse" con núcleo de la red semántica para los hombres universitarios del área de Ciencias de la Salud, lo que podría relacionarse con el área de formación de estos, ylo que supone hipotéticamente una preocupación mayor por la alimentación, debido a la centralidad que asume esta dimensión en el contexto de formación profesional en el que se desenvuelven.

Ahora bien, en el contexto de la sociedad de consumidores, los objetos de consumo, como la comida y la alimentación, representan mucho más que ingerir y digerir, por lo que se incluye en el plano de significado el placer que esto produce (Bauman, 2014). Es más: el cuerpo del consumidor es una fuente fecunda de ansiedad inacabable, movido por un consumismo que gira en torno a la incitación de deseos siempre nuevos, deseos amparados por un idealfitness. Si bien los hombres han puesto tradicionalmente énfasis en la funcionalidad del cuerpo, en la actualidad prestan más atención al aspecto corporal, a la salud física y a la emocional (Rosenmann et al., 2017). Por lo tanto, la palabra "alimentarse", como núcleo de la red semántica, podría relacionarse con la preocupación por el aspecto corporal en los hombres universitarios. Además, el vínculo entre la alimentación y la construcción de identidad masculina se releva en los análisis del problema, en los que, por ejemplo, el consumo de carne se ha interpretado como un hábito alimentario que es símbolo de consumo conspicuo, y que puede ser considerado como un símbolo de masculinidad (Stibbe, 2012).

Por otra parte, la definición de "consumo" a partir de palabras como "gastos", "dinero" y "comprar", ubicados en los primeros lugares de la red semántica general, implica una consideración de consumo asociada al intercambio a partir de la adquisición de bienes materiales o inmateriales. En ese sentido, Duarte (2009), en una investigación con jóvenes chilenos, evidenció la importancia del consumo material (opulento) como mecanismo para lograr el éxito en jóvenes empobrecidos. Por otra parte, Dittmar (2005) afirma que los valores fundamentales de la sociedad de consumo son materiales, lo que es un hecho manifiesto si se considera el alto porcentaje de endeudamiento juvenil en Chile, fenómeno que aumenta sostenidamente a través del tiempo (INJUV, 2017). Esto demuestra que la adquisición de bienes de consumo cumple un rol central en la sociedad de consumidores (Bauman, 2014 y 2016), en la sociedad del hiperconsumo (Lipovetsky y Serroy, 2010), y es el valor de la novedad lo que prima por sobre lo perdurable, incluso para sociedades encaminadas a niveles de desarrollo más emergentes. Considerando la variable género, el significado de consumo asociado a gastos, dinero y comprar de los hombres entrevistados son coherentes con 
los reportes de Barros et al. (2019); Cubillas et al. (2016); Eastman et al. (2018); y Kim y Jung (2014), que indican la valoración de los hombres a las posesiones materiales, lo que puede ser relacionado con el rol de proveedor económico presente en sus construcciones de masculinidades.

Cabe señalar que el contexto chileno, asentado sobre el modelo económico neoliberal, es un ambiente fructífero para el consumo, por lo que se transforma en un agente ambivalente de individuación (Araujo y Martuccelli, 2012). De este modo, el consumo como práctica social genera placer y satisfacción, pero además provoca culpa e insatisfacción. Esto puede evidenciarse en la definición que construyen respecto del consumo hombres jóvenes, a partir de las palabras definitorias como "innecesario" y "satisfacción", contenidos, como se ha indicado, en la red semántica general; hallazgos coincidentes con los reportados por Barros et al. (2015).

Por otra parte, las palabras "drogas", "alcohol", "dependencia", “tomar"/“beber", "vicios", presentes en la red semántica de los informantes, indican un rol protagónico de los significados asociados al consumo en estos jóvenes. Cabe mencionar que, en el caso de estudiantes de Ciencias de la Salud, la existencia de palabras definitorias de consumo en relación con el alcohol y las drogas se encuentra en los primeros lugares de la red, a diferencia de los estudiantes de las Ciencias Sociales y Ciencias de la Ingeniería. Ahora bien, esto no es un resultado inesperado si se considera que el consumo de drogas y alcohol es un fenómeno relevante en Chile. Solo considerando el consumo de marihuana, se aprecia un aumento significativo, de un 24 $\%$ en 2014 a un $33.8 \%$ en el 2016, en el grupo de jóvenes de entre 19 a 25 años. Asimismo, cabe señalar que el consumo de alcohol en el mismo tramo de edad llegó a un $56.7 \%$ hace pocos años, siendo mayor el porcentaje en varones (SENDA, 2017). Los hallazgos anteriores, con ayuda de antecedentes ya existentes, muestran que es posible considerar el vínculo entre la masculinidad y el consumo de alcohol, a partir de la evidencia que sugiere que el uso de alcohol en jóvenes tiende a transformarse en una estrategia de validación y reconocimiento entre pares (Toquero y Sarguero, 2013; Vásquez y Castro, 2009).

La palabra "ropa" forma parte de la definición de consumo de los hombres universitarios, presente en el conjunto SAM de los estudiantes de Ciencias de la Salud. En ese sentido, la apariencia se ha reportado como un aspecto central para los hombres jóvenes (Fuller, 2018; Villa, 2015), más aún en el contexto de sociedad de consumidores, en el que los objetos materiales adquieren centralidad junto al estilo, y pueden asociarse a las pretensiones de éxito (McNeill y McKay, 2016). Los hombres jóvenes, hoy, parecen estar poniendo más atención a este espacio atribuido tradicionalmente a las mujeres (McNeill y McKay, 2016). Al respecto, Pallavicini (2008) destaca la búsqueda de la personalización que se da a través del consumo de objetos en serie y de moda. Cabe señalar que, en esta misma línea, Isaksen y Roper $(2008,2012)$ revelaron que las marcas son fundamentales para la formación y expresión de la identidad de los adolescentes. De esta forma, la vestimenta y el modo en que se cubre el cuerpo masculino constituyen construcciones sociohistóricas que comunican información sobre los sujetos (Enguix, 2013) y que constituyen expresiones de sus masculinidades.

Como limitación de este estudio puede señalarse el número reducido de la muestra, lo que implica que el carácter intencionado para conformarla permite remitir los resultados solo a la muestra seleccionada y sus sujetos. Sin embargo, este estudio constituye una primera aproximación a la relación entre el consumo y los hombres jóvenes, y aporta a la identificación áreas de indagaciones futuras. Respecto de los temas emergentes, se identifica el consumo de los alimentos en hombres. Otra área de indagación propuesta muestra la asociación del consumo con las 
palabras "gastos", "dinero" y "comprar", en relación con la valoración de la materialidad y la centralidad que tiene el consumo y las prácticas de consumo para la exploración identitaria de hombres jóvenes. El consumo de drogas y alcohol, asimismo, es un ámbito de exploración de interés para el grupo etario en cuestión, razón por la cual puede considerarse una preocupación recurrente en términos de investigación empírica.

A la luz de los resultados de este estudio, la relación entre las masculinidades y el consumo resulta un desafío investigativo interesante, considerando los contextos culturales, económicos y sociales particulares de un espacio regional, el cual ejemplifica lo que puede estar sucediendo en otros planos y contextos de la sociedad actual. A esto se añade el fenómeno de las juventudes, ambos analizados como espacios de articulación entre prácticas, sentidos y valores (Elizalde, 2018). Los estudios sobre masculinidades, como un marco necesario de exploración para comprender el consumo y sus prácticas en nuestras sociedades, emergen como un campo de abordaje empírico por parte de las ciencias sociales actuales, para las cuales los grupos sociales emergentes, como los jóvenes, asumen un interés difícil de encontrar en tiempos anteriores.

\section{Referencias}

Álvarez, C. y Garcés, A. (2017). La construcción de generación en los discursos juveniles del Chile actual. Revista Latinoamericana de Ciencias Sociales, Niñez y Juventud, 15(2), 991-1004. https://doi.org/10.11600/1692 715x.152130310201616

Alexander, S. (2003). Stylish hard bodies: Branded masculinity in "men's health" magazine. Sociological Perspectives, 46(4), 535-554. https:// doi.org/10.1525/sop.2003.46.4.535

Araujo, K. y Martuccelli, D. (2012). Desafios comunes. Retrato de la sociedad chilena. Santiago: LOM Ediciones.

Arnett, J. (2007). Emerging adulthood: What is it, and what is it good for? Child Development Perspectives, 1(2), 68-73. https://doi. org/10.1111/j.1750-8606.2007.00015.x

Arnett, J. (2015). Emerging Adulthood. Nueva York: Oxford University Press.

Bakewell, C. y Mitchell, V. (2003). Generation Y. Female consumer decision-making styles. International Journal of Retail \& Distribution Management, 31(2), 95-106. https://doi. org/10.1108/09590550310461994

Barros, S., Cárdenas, V. y Denegri, M. (2015). Concepciones y prácticas de consumo y endeudamiento en adolescentes de la ciudad de Temuco, Chile. Revista IIPSI, 18(1), 17-28. https://doi.org/10.15381/rinvp.v18i1.11770

Barros, S., Denegri, M. y Salazar, P. (2019). Consumo, actitudes hacia el endeudamiento, materialismo e influencia de pares en adolescentes rurales del sur de Chile. Interdisciplinaria, 36(1), 203-219. https://doi.org/10.16888/ interd.36.1.14

Baudrillard, J. (2007). La sociedad de consumo. Sus mitos, sus estructuras. Madrid: Siglo XXI Editores.

Bauman, Z. (2014). Vida líquida. Barcelona: Paidós.

Bauman, Z. (2016). Vida de consumo. México: Fondo de Cultura Económica.

Belk, R. y Costa, J. (1998). The mountain man myth: A contemporary consuming fantasy. Journal of Consumer Research, 25(3), 218-40. https://doi.org/10.1086/209536

Borrás, V. (2008). Las desigualdades en el consumo a través del género. Revista Española De Sociología, (8), 140-156. https://recyt. fecyt.es/index.php/res/issue/view/3300

Botero, M., Gutiérrez De Piñeres, M., Manjarrés, L. y Torres, T. (2008). La relación del SELF con el consumo en hombres y mujeres entre 18 y 24 años, estudiantes de la Universidad del Norte. Psicología desde el Caribe, 21, 1-31.

Castellanos L., Sepúlveda, J. y Denegri, M. (2016). Análisis Teórico de la relación entre estilos de compra, valores materiales y satisfacción con la vida en la adolescencia. Revista de Psicología y Ciencias del Comportamiento, 7(1), 1-22. https://doi.org/10.1804/rpcc-uacjs. 
v7i1.126

Chomsky, N. (1977). El lenguaje y el entendimiento. Barcelona: Seix Barral.

CNED. (2019). Matrícula Sistema de Educación Superior. https://www.cned.cl/indices/matricula-sistema-de-educacion-superior

CNTV y McCann, E. (2005). Informe 13.17 Adolescentes Chilenos. https://www.cntv.cl/ informe-13-17-adolescentes-chilenos-2005/ cntv/2011-04-14/180430.html

Connell, R. (1997). La organización social de la masculinidad. En Valdés, T. y Olavarría, J. (Eds.), Masculinidad/es: poder y crisis, (pp. 31-48). Santiago, Chile: Ediciones de las Mujeres.

Connell, R. (2003). Adolescencia en la construcción de masculinidades contemporáneas. En Olavarría, J., Varones adolescentes: género, identidades y sexualidades en América Latina, 71-83. Santiago: FLACSO.

Creswell, J. (2007). Qualitative inquiry and research design. Estados Unidos: Sage.

Cubillas, M., Abril, E., Domínguez, S., Román, R., Hernández, A. y Zapata, J. (2016). Creencias sobre estereotipos de género de jóvenes universitarios del norte de México. Diversitas: perspectivas en Psicología, 12(2), 217-230. https://doi.org/10.15332/s17949998.2016.0002.04

Denegri, M., Barros, S., Cárdenas, V., Sepúlveda, J. y Vivallo, O. (2017). Consumo y endeudamiento en adolescentes escolarizados de La Región de La Araucanía, Chile. Estudios Pedagógicos, 43(1), 61-74. https://doi. org/10.4067/S0718-07052017000100004

Denegri, M., García, C., González, N. y Sepúlveda, J. (2014). ¿Educadores o consumidores? Discrepancia del yo, Consumo y valores materiales en estudiantes chilenos de pedagogía. Revista Electrónica Actualidades Investigativas en Educación, 14(2), 1-29.

Dittmar, H. (2005). Compulsive buying-a growing concern? An examination of gender, age, and endorsement of materialistic values as predictors. British Journal of Psychology, 96, 467-491. https://doi.org/10.1348/000712605X53533

Dittmar, H. (2011). Material and consumer iden- tities. En S. J. Schwartz, K. Luyckx y V. L. Vignoles (Eds.), Handbook of identity theory and research (pp. 745-769). New York: Springer

Duarte, C. (2009). Sobre los que no son, aunque sean. Éxito como exclusión de jóvenes empobrecidos en contextos capitalistas. Última década, 17(30), 11-39. https://doi.org/10.4067/ S0718-22362009000100002

Eastman, J., Iyer, R., Shepherd, C., Heugel, A. y Faulk, D. (2018). Do they shop to stand out or fit in? The luxury fashion purchase intentions of young adults. Psychology \& Marketing, 35, 220-236. https://doi.org/10.1002/mar.21082

Elizalde, S. (2018). Contextos que hablan. Revisiones del Vínculo Género/Juventud: del caso María Soledad al \#Niunamenos. Última Década, 26(50), 157-179. https://doi. org/10.4067/S0718-22362018000300157

Eng, T. Y. y Bogaert, J. (2010). Psychological and cultural insights into consumption of luxury western brands in India. Journal of Customer Behaviour, 9(1), 55-75. https://doi. org/10.1362/147539210X497620

Enguix, E. (2013). Cuerpos desbordados: La construcción corporal de la masculinidad. Argos, 30(59), 060-086.

Figueroa, J., González, E. y Solís, V. (1981). Una aproximación al problema del significado: las redes semánticas. Revista Latinoamericana de Psicología, 13(3), 447-458.

Fuller, N. (2018). El cuerpo masculino como alegoría y como arena de disputa del orden social y de los géneros. En N. Fuller (Ed.). Dificil ser hombre. Nuevas masculinidades latinoamericanas (pp. 25-45). Lima: PUCP.

Godoy, M. P., Sepúlveda, J., Araneda, L., Canario, R., Fonseca, J. y Sáez, C. (2018). Actitud hacia el consumo, ahorro y endeudamiento en titulados de una universidad pública del sur de Chile. Interdisciplinaria, 35(2), 511-525. https://doi.org/10.16888/interd.2018.35.2.16

González, M., Macías, A. y Gonzálvez, J. (2016). Consumo y género en los/as jóvenes universitarios; nuevas formas de interpretar la desigualdad social. En D. Carbonero, E. Raya, N. Caparros y C. Gimeno. (Coords.) 
Respuestas transdisciplinares en una sociedad global. Aportes desde el Trabajo Social (pp. 1-20). Logroño, España: Universidad de La Rioja.

Hofstede, G. (1980). Culture's consequences: International differences in workrelated values. Beverly Hills, CA: Sage.

Huber, L. (2002). Consumo, cultura e identidad en el mundo globalizado: estudios de caso en los Andes. Lima: IEP.

Isaksen, K. y Roper, S. (2008). The Impact of Branding on Low-Income Adolescents: A Vicious Cycle? Psychology \& Marketing, 25(11): 1063-1087. https://doi.org/10.1002/ mar.20254

Isaksen, K. y Roper, S. (2012). The Commodification of Self-Esteem: Branding and British Teenagers. Psychology \& Marketing, 29(3), 117-135. https://doi.org/10.1002/mar.20509

INJUV (2017). Octava Encuesta Nacional de la Juventud, 2015. Santiago: Ministerio de Desarrollo Social.

Kim, D. y Jang, S. (2014). Motivational drivers for status consumption: A study of Generation Y consumers. International Journal of Hospitality Management, 38, 39-47. https://doi. org/10.1016/j.ijhm.2013.12.003

Lipovetsky, G. (2007). La felicidad paradójica. Ensayo sobre la sociedad de hiperconsumo. Barcelona: Anagrama.

Lipovetsky, G. y Serroy, J. (2010). La cultura-mundo. Respuesta a una sociedad desorientada. Barcelona: Anagrama.

Littlefield, J. y Ozanne, J. (2011). Socialization into consumer culture: hunters learning to be men. Consumption Markets \& Culture, 14(4), 333-360. https://doi.org/10.1080/10253866.20 11.604494

Maguire, J. S. y Stanway, K. (2008). Looking good: Consumption and the problems of self-production. European Journal of Cultural Studies, 11(1), 63-81. https://doi. org/10.1177/1367549407084964

McNeill, L. y McKay, J. (2016). Fashioning masculinity among young New Zealand men: young men, shopping for clothes and social identity, Young Consumers, 17(2), 143-154.
https://doi.org/10.1108/YC-09-2015-00558

MINEDUC (2019). Gratuidad. Subsecretaria de Educación Superior. Ministerio de Educación, Chile. http://www.gratuidad.cl/lo-que-debessaber/

Olavarría, J. (2000). De la identidad a la política: masculinidades y políticas públicas. Auge y ocaso de la familia nuclear patriarcal en el siglo XX. En J. Olavarría y R. Parrini (Eds.), Masculinidad/es. Identidad, sexualidad y familia (pp. 11-28). Santiago: LOM.

Olavarría, J. (2018). Masculinidades. Paternidades y Familias. ¿Qué es lo que viene? En N. Fuller (Ed.), Dificil ser hombre. Nuevas masculinidades latinoamericanas (pp. 85-108). Lima: Pontificia Universidad Católica del Perú.

Ortega, V. y Rodríguez, J. (2005). Consumo y deuda en adultos jóvenes: evaluación desde un modelo integrador de la conducta económica. Revista Latinoamericana de Psicología, 37(1), 95-118.

Pallavicini, P. (2008). Los objetos de consumo en la construcción de los procesos de individuación de adolescentes. Última Década, 16, 29-46.

Pérez, L. (2014). El peso real de la deuda de estudios: La problemática de los jóvenes deudores del sistema de financiamiento universitario de la Corfo pregrado en Santiago de Chile. Archivos Analíticos de Politicas Educativas, 22(75). https://doi.org/10.14507/epaa. v22n75.2014

PNUD/INJUV. (2003). Transformaciones culturales e identidad juvenil en Chile. Santiago, Chile: PNUD/INJUV.

Rosenmann, A., Kaplan, D., Gaunt, R., Pinho, M. y Guy, M. (2017). Consumer masculinity ideology: Conceptualization and initial findings on men's emerging body concerns. Psychology of Men \& Masculinity, 19(2), 257-272. https:// doi.org/10.1037/men0000095

Ruiz, J. (2012). Metodología de la Investigación Cualitativa. Bilbao: Universidad de Deusto.

Sassatelli, R. (2012). Consumo, cultura y sociedad. Buenos Aires: Amorrortu Editores.

Schnettler, B., Miranda, H., Sepúlveda, J. y Denegri, M. (2011). Satisfacción con la 


\section{Arias Lagos, Denegri Coria y Sáez Ardura}

alimentación y la vida, un estudio exploratorio en estudiantes de la Universidad de La Frontera, Temuco-Chile. Psicología \& Sociedad, 23(2), 426-435. https://doi.org/10.1590/S010271822011000200024

SENDA (2017). Décimo Segundo Estudio de Drogas en Población General 2016. Santiago: Gobierno de Chile. http://www.senda. gob.cl/wp-content/uploads/2017/12/InformeENPG2016.pdf

Stibbe, A. (2012). Advertising, gender and health advice: The case of men's health in the year 2000. Masculinities \& Social Change, l(3), 190-209. https://doi.org./10.17583/ msc. 2012.329

Toquero, M. y Salguero, M. (2013). Los significados de ser hombre asociados al consumo de sustancias psicoactivas. La Ventana. Revista de estudios de género, 4(38), 372-404. https://doi. org/10.32870/lv.v4i38.537

Valdez, J. (1998). Las Redes semánticas naturales. Usos y aplicaciones en Psicología Social. México: Universidad Autónoma del Estado de México.

Valles, M. (2002). Entrevistas Cualitativas. Madrid: Centro de Investigaciones Sociológicas.

Vázquez, V. y Castro, R. (2009). Masculinidad hegemónica, violencia y consumo de alcohol en el medio universitario. Revista Mexicana de Investigación Educativa, 14(42), 701-719.

Vera, J., Pimentel, C. y Batista, F. (2005). Redes semánticas: Aspectos teóricos, técnicos, metodológicos y analíticos. Ra Ximhai, 3(1), 439-451.

Villa, J. (2015). Cuerpo, masculinidad y estilo en jóvenes de sectores altos de Lima. Debates en Sociología, 40, 61-91.

Recibido: 23 de mayo de 2020 Aceptado: 10 de septiembre de 2021 